\title{
Photothermoelectric response in asymmetric carbon nanotube devices exposed to sub- terahertz radiation
}

Cite as: Appl. Phys. Lett. 103, 181121 (2013); https://doi.org/10.1063/1.4828555

Submitted: 09 August 2013. Accepted: 23 September 2013. Published Online: 01 November 2013

G. Fedorov, A. Kardakova, I. Gayduchenko, I. Charayev, B. M. Voronov, M. Finkel, T. M. Klapwijk, S. Morozov, M. Presniakov, I. Bobrinetskiy, R. Ibragimov, and G. Goltsman
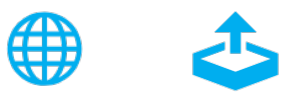

\section{ARTICLES YOU MAY BE INTERESTED IN}

Response of asymmetric carbon nanotube network devices to sub-terahertz and terahertz radiation

Journal of Applied Physics 118, 194303 (2015); https://doi.org/10.1063/1.4935947

Terahertz detection in single wall carbon nanotubes

Applied Physics Letters 92, 033105 (2008); https://doi.org/10.1063/1.2837188

Dual origin of room temperature sub-terahertz photoresponse in graphene field effect transistors

Applied Physics Letters 112, 141101 (2018); https://doi.org/10.1063/1.5018151

\section{Challenge us.}

What are your needs for periodic signal detection?

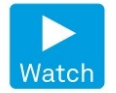

Zurich

- Instruments

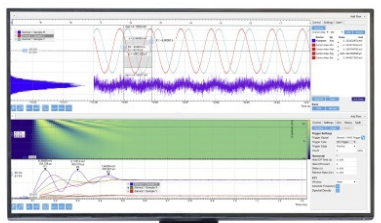

. 


\title{
Photothermoelectric response in asymmetric carbon nanotube devices exposed to sub-terahertz radiation
}

\author{
G. Fedorov, ${ }^{1,2, a)}$ A. Kardakova, ${ }^{2,3}$ I. Gayduchenko, ${ }^{1}$ I. Charayev, ${ }^{3}$ B. M. Voronov, ${ }^{3}$ \\ M. Finkel, ${ }^{3}$ T. M. Klapwijk, ${ }^{3,4}$ S. Morozov, ${ }^{2}$ M. Presniakov, ${ }^{1}$ I. Bobrinetskiy, ${ }^{5}$ R. Ibragimov, ${ }^{5}$ \\ and G. Goltsman 3,6 \\ ${ }^{1}$ National Research Centre "Kurchatov Institute," Moscow 123128, Russia \\ ${ }^{2}$ Moscow Institute of Physics and Technology, State University, Dolgoprudny 141700, Russia \\ ${ }^{3}$ Physics Department, Moscow State Pedagogical University, Moscow 119991, Russia \\ ${ }^{4}$ Kavli Institute of Nanoscience, Delft University of Technology, 2600 AA Delft, Netherlands \\ ${ }^{5}$ National Research University of Electronic Technology (MIET), Zelenograd 124498, Russia \\ ${ }^{6}$ Moscow Institute of Electronics and Mathematics, National Research University Higher School of Economics, \\ Moscow 109028, Russia
}

(Received 9 August 2013; accepted 23 September 2013; published online 1 November 2013)

\begin{abstract}
We report on the voltage response of carbon nanotube devices to sub-terahertz (THz) radiation. The devices contain carbon nanotubes (CNTs), which are over their length partially suspended and partially Van der Waals bonded to a $\mathrm{SiO}_{2}$ substrate, causing a difference in thermal contact. We observe a DC voltage upon exposure to $140 \mathrm{GHz}$ radiation. Based on the observed gate voltage and power dependence, at different temperatures, we argue that the observed signal is both thermal and photovoltaic. The room temperature responsivity in the microwave to $\mathrm{THz}$ range exceeds that of CNT based devices reported before. (C 2013 AIP Publishing LLC. [http://dx.doi.org/10.1063/1.4828555]
\end{abstract}

The unique band structure of carbon nanotubes (CNT) makes them good candidates for optoelectronic applications in a wide frequency range. ${ }^{1-3}$ Due to the quantization of the electron wave vector around the circumference a CNT with $n-m \neq 3 i$, where $i$ is an integer is a semiconductor with an energy gap $\varepsilon_{g} \sim 1 / r$ (with $r$ the radius of the CNT). ${ }^{4}$ The optical properties of semiconducting nanotubes, in the visible and near infrared range, have been intensely studied during the last ten years. ${ }^{1,5,6}$ It has been shown that they can be used as basic elements for different optoelectronic functions, such as photodetectors ${ }^{6}$ or light emitting diodes. ${ }^{7}$ Nanotubes, with $n-m=3 i$, with $n \neq m$, are called quasi-metallic, due to their small band gap, which ranges from about 1 to about $30 \mathrm{meV}$ for nanotubes with a diameter of $1-2 \mathrm{~nm}^{8,9}$ Socalled armchair CNTs, with $n=m$, are truly metallic. Quasi-metallic CNTs are potentially very good candidates for optoelectronics in the $\mathrm{THz}$ range because their bandgaps fall into the corresponding energy range.

Previous research on $\mathrm{THz}$ detectors based on CNTs has brought interesting new results. ${ }^{3,10}$ Diode type detection and bolometric detection were reported in Refs. 3 and 10. Photonassisted tunneling in the Coulomb blockade regime was reported in Ref. 11. The response of antenna-coupled fully suspended single carbon nanotubes to sub- $\mathrm{THz}$ radiation was reported in Ref. 12. A power-dependent DC voltage was reported in this work and interpreted as a combined effect of the increased temperature of the electron liquid with respect to the electrodes and requiring an asymmetry in the contact resistances.

Here we report on CNT devices, which will have a temperature gradient due to a difference in heat transfer along the CNTs while exposed to radiation. The different heat transfer conditions occur because of a difference in coupling to the substrate along the length. The devices show a DC

\footnotetext{
${ }^{\text {a) }}$ Author to whom correspondence should be addressed. Electronic mail: gefedorov@mail.ru
}

voltage signal in response to radiation in the sub- $\mathrm{THz}$ range in the temperature range from 4.2 to $300 \mathrm{~K}$. We argue that the room temperature response is thermal in origin, similarly to the photoresponse of CNT films reported before, e.g., in Ref. 13. The data obtained at low temperatures indicate contributions of both thermal effects and of photovoltaic effects. While it is not clear if the observed effect can be used to develop efficient $\mathrm{THz}$ detectors we note that the responsivity of our devices exceeds that of CNT based devices in microwave or $\mathrm{THz}$ range reported before (even at room temperature). Besides at $4.2 \mathrm{~K}$ notable increase of the sample conductance (at least four-fold) is observed.

The nanotubes are grown using the CVD method, following the procedures described in Ref. 14. The catalyst islands, containing $\mathrm{Fe}\left(\mathrm{NO}_{3}\right)_{3} \cdot 9 \mathrm{H}_{2} \mathrm{O}, \mathrm{MoO}_{2}(\mathrm{acac})_{2}$ and alumina $\left(\mathrm{Al}_{2} \mathrm{O}_{3}\right)$ nanoparticles, are defined by e-beam lithography and patterned by lift-off of a methacrylic acid (MAA)/Poly(methyl methacrylate) (PMMA) bi-layer. The substrate is boron-doped silicon covered with $250 \mathrm{~nm}$ of thermally grown $\mathrm{SiO}_{2}$. The room temperature resistivity of the silicon is $10 \Omega \cdot \mathrm{cm}$, which is practically transparent for radiation in the used frequency range. The conductivity, even down to liquid helium temperatures, is high enough to use it as a backgate. The size of the substrates is 4 by $4 \mathrm{~mm}^{2}$. The catalyst islands are located close to predefined alignment marks (Fig. 1(a)). After the CVD growth of the CNTs they are imaged with a scanning electron microscope (SEM) to identify their position with respect to the alignment marks. Subsequently, metal electrodes are formed, using lift-off.

As shown in Figs. 1(b) and 1(c) the area of the catalyst island is covered with a relatively dense network of CNTs. Most of the CNTs are sticking out of the catalyst islands and are therefore suspended, i.e., not in direct contact with the substrate. Some CNTs are directed to extend far outside the area of the catalyst islands. These CNTs on the $\mathrm{SiO}_{2}$-surface are attracted to the substrate by Van-der-Waals forces. 
(a)

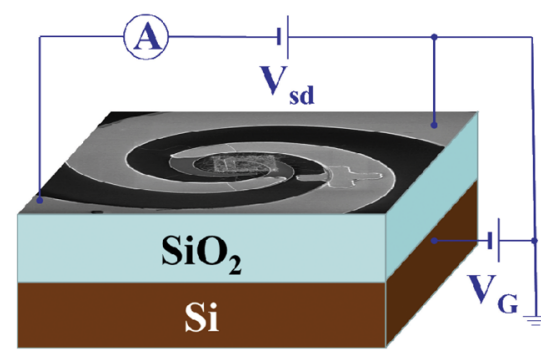

(c)

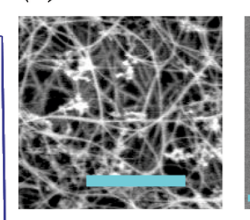

(d)

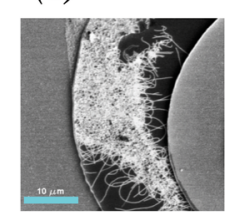

(e)
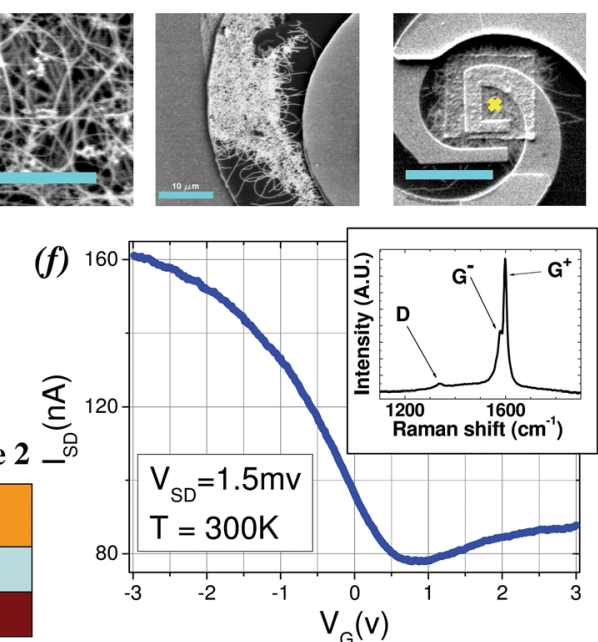

(b)

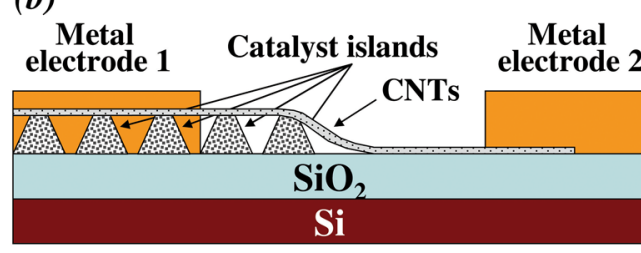

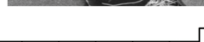

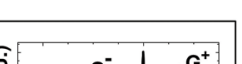

FIG. 1. (a) Schematic diagram of the measurement circuit. (b) Schematics representation of the sensing element illustrating the current path from on electrode to another going from mostly suspended CNTs to those attached to the substrate. (c) Scanning electron microscopy (SEM) image of the CNT film inside the catalyst island; scale bar is $0.5 \mu \mathrm{m}$. (d) SEM image of device D1; scale bar is $10 \mu \mathrm{m}$. (e) SEM images of device D2; scale bar is $10 \mu \mathrm{m}$; the cross indicates position in which the Raman spectrum was measured. (f) Transfer characteristic of device $\mathrm{D} 2$. The inset shows the Raman spectrum measured in the spot labeled with a cross in the image (e).
The devices are coupled to the radiation with a logarithmic spiral antenna, ${ }^{15}$ which also serves for DC contacts (Fig. 1(a)). They are made in such a way that one of the electrodes makes contact to the dense CNT-network, partially overlapping the catalyst island, while the other one contacts the few CNTs located outside this island. We will present results obtained with in two samples referred to as D1 and D2 (see Figs. 1(d) and 1(e)). Besides the unavoidable differences in dimensions the electrodes of two samples are also different. For sample D1 the CNTs are contacted with $100 \mathrm{~nm}$ thick aluminum film with a thin ( $2 \mathrm{~nm}$ thick) adhesion layer of chromium. For sample D2 the CNTs are contacted with a $70 \mathrm{~nm}$ thick gold film on a $2 \mathrm{~nm}$ adhesion layer of chromium.

The CNTs are characterized by measuring their Raman spectra. A typical measured spectrum is shown in the inset to Fig. 1(f). From the ratio of the $\mathrm{G}^{+}$and $\mathrm{G}^{-}$peak amplitudes we conclude that about $1 / 3$ of our CNTs are metallic or quasi-metallic. ${ }^{16}$ The observation of radial breathing mode peaks observed between 120 and $140 \mathrm{~nm}$ (not shown) points towards single walled CNTs with diameters between 1.5 and $2.3 \mathrm{~nm}$. The fraction of metallic CNTs can also be estimated using the transfer characteristics of our devices (Fig. 1(f)). The ratio of the OFF and ON currents equals $0.4 \pm 0.1$ consistent with the Raman data. The enhanced conduction at negative gate voltage indicates that the CNTs are p-doped.

The experimental set-up is shown in Fig. 2(a). The silicon substrates with the samples are fixed on a flat surface of a silicon lens, placed inside the cryostat, equipped with PTFE optical window. Measurements were carried out at two temperatures: $300 \mathrm{~K}$ and $4.2 \mathrm{~K}$. The sensing element of the device, the CNTs inside the antenna, is located in the focus of the lens, enabling good coupling to the incident electromagnetic radiation. The power incident on the cryostat window is measured with a Golay cell. The combined optical losses at the PTFE window, the lens, etc. are estimated to be approximately $3 \mathrm{~dB}$ and are caused primarily by the reflection losses at the Si lens surface. A backward wave oscillator (BWO) is used as a source of sub- $\mathrm{THz}$ radiation with a frequency of $140 \mathrm{GHz}$. The maximum power of the radiation at the cryostat window was $800 \mu \mathrm{W}$ and was adjusted by a wire-grid attenuator. A voltage $V_{G}$ can be applied between the substrate and one of the antenna electrodes. All measurements at a fixed gate voltage $V_{G}$ are carried out with the gate shorted to the ground $\left(V_{G}=0\right)$. The response voltage across the nanotubes $V_{\text {resp }}$ is measured using a lock-in amplifier while radiation is amplitudemodulated with a modulation frequency varied from $100 \mathrm{~Hz}$ to $100 \mathrm{kHz}$. The I-V characteristics of the sample are measured using a Keithley 2400 sourcemeter.

The IV-curves measured at room temperature are fully linear (Figs. 1(b) and 1(c)). For sample D1 the resistance is about $200 \mathrm{k} \Omega$ while for D2 the resistance is about $15 \mathrm{k} \Omega$. Focusing first on sample D2 (Fig. 1(c)) at room temperature, we observe that exposed to radiation with a power of $800 \mu \mathrm{W}$ the IV curve shifts to the left so that the current at zero bias voltage equals about $0.1 \mu \mathrm{A}$. The zero-current crossing occurs at a bias voltage of $-2 \mathrm{mV}$. This clearly indicates the appearance of a DC voltage induced by the (a)

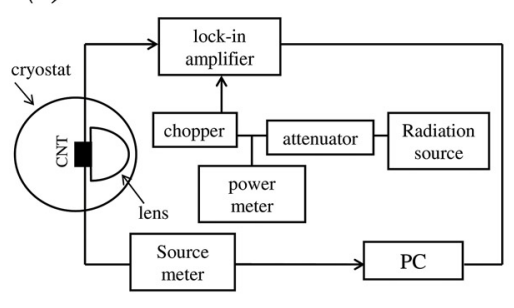

(b)

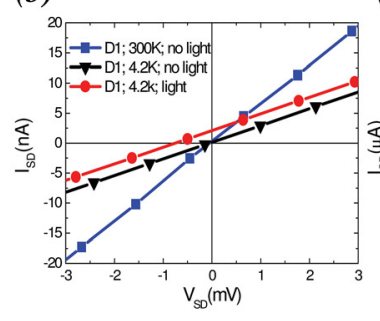

(c)

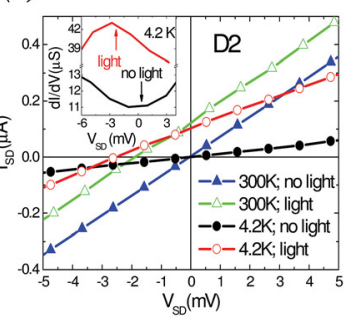

FIG. 2. (a) The general scheme of the experimental setup for recording the response of devices based on carbon nanotubes for THz radiation. IV-curves of D1 (b) and D2 (c). Room temperature dark current as a function of bias voltage compared to dark and photo current as a function bias voltage at $4.2 \mathrm{~K}$. The inset in (c) shows the differential conductance as function of bias voltage at $4.2 \mathrm{~K}$ with and without radiation. 
radiation, which we further refer to as the response voltage $V_{\text {resp }}$. At this temperature no significant change in differential conductance, caused by the radiation, is observed. A similar response to the radiation is observed for sample D1 although the magnitude of the response is much smaller (about $50 \mu \mathrm{V}$ for maximum radiation power) and detectible only with the aid of the phase-sensitive lock-in technique.

As the temperature is decreased the resistance of both samples goes up presumably due to suppression of conductance of the semiconducting nanotubes. At liquid helium temperatures the IV characteristic of sample D1 remains linear although the resistance increases to $330 \mathrm{k} \Omega$. Also, in this case the radiation induces a DC voltage with a larger value of $550 \mu \mathrm{V}$ at the maximum power of radiation, accompanied by a small decrease in resistance by about $1 \%$. The sample D2 shows at liquid helium temperatures a nonlinear IV curve. The differential conductance reaches its minimum at zero source-drain voltage (see the inset of Fig. 2(c)). Exposed to radiation we find again a DC shift to negative voltages of about $3 \mathrm{mV}$, accompanied by a considerable increase in conductivity by a factor of 3-4. Upon exposure to radiation the non-linearity changes also. The conductance minimum becomes a maximum at a negative voltage of $3 \mathrm{mV}$, roughly coinciding with the zero-crossing of the IV curve. This qualitative change in non-linearity is currently not well understood, and we focus further on the main observation: the radiation-induced DC voltage and the change in conductivity-level.

In both samples the response at room temperature increases linearly with power (Fig. 3(a)). The overall lower response level in sample D1 is most likely due to its larger resistance $\mathrm{R}$, which is much larger than the antenna impedance $Z_{0}$. In this case the power delivered to the CNTs is inversely proportional to the device resistance. The response of sample D1 is about 20 times weaker than that of device
D2, consistent with a difference in device resistance by a factor of 16.

At liquid helium temperatures, $4.2 \mathrm{~K}$, the $\mathrm{V}_{\text {resp }}$ dependence on radiation power $\mathrm{P}$ is no longer linear for both devices and in fact shows a region where the sign of the response is not but positive initially negative. Only for powers larger than $70 \mu \mathrm{W}$ the sign of the DC voltage shift is analogous to what is observed at room temperature. At helium temperature $\mathrm{V}_{\text {resp }}$ has opposite sign for incident powers below $70 \mu \mathrm{W}$ in case of D1 and below $25 \mu \mathrm{W}$ in case of D2 (see the inset of Fig. 3(a)). The fact that we observe this effect in both devices is an indication that it is generic.

As a check on the measurement-method we measured $V_{\text {resp }}$ at different modulation frequencies $F$ of the lock-in. Fig. 3(b) shows the observed $V_{\text {resp }}(F)$ curves. If we define the response time $\tau_{\text {resp }}$ of the device by fitting with $V_{\text {resp }}=\frac{V_{0}}{\sqrt{1+\tau^{2} \omega^{2}}}$, we find a response time $\tau_{\text {resp }}=120 \mu$ s for the sample D1 and $\tau_{\text {resp }}=9 \mu$ s for sample D2. Both values are in fair agreement with the product of device resistance and geometrical capacitance between the electrodes and the gate.

Finally, we measured the response for different gate voltages (Fig. 3(c)) for sample D2. We observe at room temperature, that the signal goes down for all radiation-power levels when the gate voltage is scanned from $-3 \mathrm{~V}$ to $+3 \mathrm{~V}$. At liquid helium temperatures the dependence on $\mathrm{V}_{\mathrm{g}}$ is not monotonous and exhibits hysteresis. The latter we attribute to the high resistivity of the silicon used for the gate. At low power level the magnitude of $\mathrm{V}_{\text {resp }}\left(\mathrm{V}_{\mathrm{G}}\right)$ changes sign in a narrow range of gate voltages close to zero.

In interpreting the results we assume that the radiation is absorbed by the charge carriers in the CNTs. The actual temperature of the charge carriers may differ from the phonontemperature and is determined by the coupling to the thermal bath, provided by the substrate and the electrical contacts,

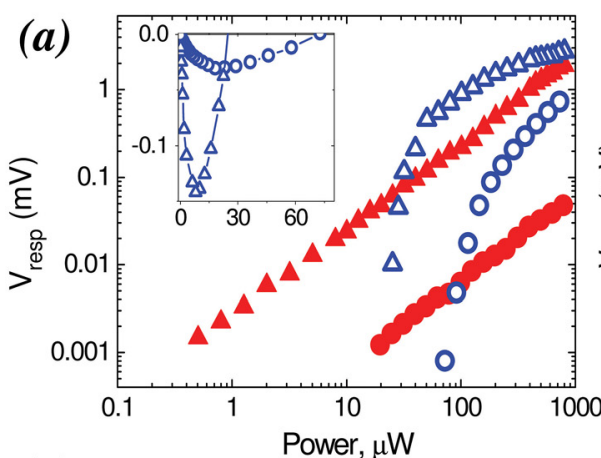

(c)

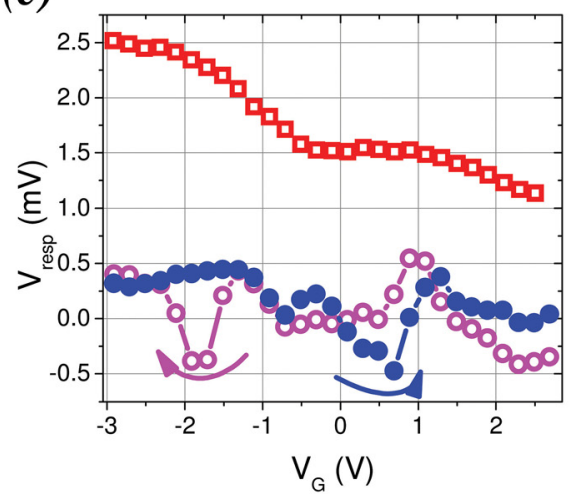

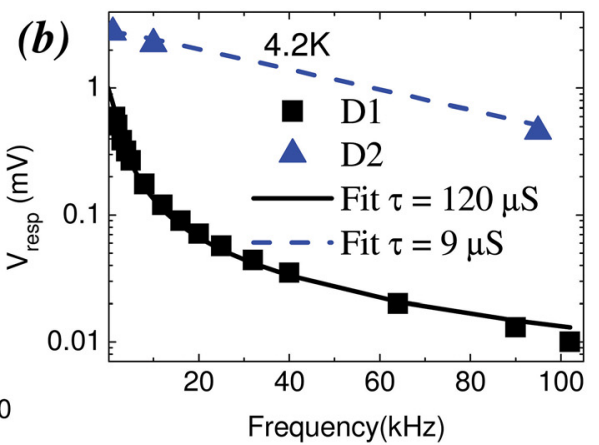

(d)

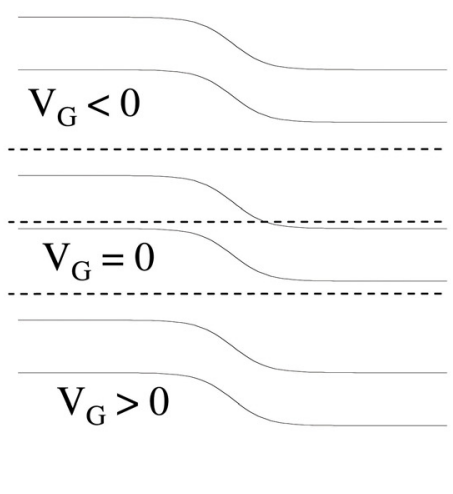

FIG. 3. (a) Response voltage as a function of incident radiation power of D1 (circles) and D2 (triangles) at room temperature (filled symbols) and $4.2 \mathrm{~K}$ (hollow symbols). The inset shows the small power area when the response voltage $V_{\text {resp }}$ is negative. (b) Response voltage as a function of the modulation frequency for the case of maximum power of incident radiation at $4.2 \mathrm{~K}$. (c) Response voltage of D2 as a function of the gate voltage $V_{G}$ at room temperature and incident power of $800 \mu \mathrm{W}$ (squares) and at $4.2 \mathrm{~K}$ and incident power of $8 \mu \mathrm{W}$ (circles). (d) Schematic band diagrams of a small gap CNT in the region of contact between suspended and non-suspended parts under different gate voltages. Dotted line shows position of the Fermi level; upper and lower solid lines show the edges of conduction and valence bands, respectively. 
which act as equilibrium reservoirs. The observed decrease of the device resistance under radiation indicates an overall heating of the CNTs. Most of the nanotubes forming the dense network on the catalyst island area are suspended and they are poorly heat sunk to the substrate. The part of the nanotubes lying on the surface of the substrates have much better thermal contact to the substrate. We can thus assume that the side of CNTs at the electrode contacting the catalyst island are heated stronger than the part contacted to the other electrode. The resulting temperature difference should give rise to the DC voltage across the device, due to the thermoelectric power (TEP) of the CNTs. Such an interpretation is consistent with previous work done on CNT films (e.g., Ref. 13), in which the film was heated locally by the laser beam.

The sign of the signal at room temperature indicates that the main type of carriers in our devices is holes, which is typical for carbon nanotubes ${ }^{17}$ and consistent with the $I\left(V_{G}\right)$ data presented in Fig. 1(f). Applying a negative gate voltage increases the number of holes and as shown in Fig. 3(c) the $V_{\text {resp }}$ signal is increased consistently with this scenario. Since the radiation provides a small temperature rise, compared to room temperature, a linear increase with incident power is plausible.

At liquid helium temperature the observed response is more complex. The change of the $V_{\text {resp }}$ sign at lower radiation power may indicate a change of sign of the thermoelectric power of some CNTs. Indeed, it has been shown before ${ }^{18}$ that the sign of the TEP of individual single-walled nanotubes is tuned by the gate voltage. Importantly, the TEP sign change with gate voltage was reported in Ref. 18 at temperatures up to $300 \mathrm{~K}$. Since our devices are made from multiple CNTs their contributions are averaged so that we are likely to detect the net response, which leads to a positive TEP at room temperature regardless of the $\mathrm{V}_{\mathrm{G}}$ value. This would imply that at low temperatures the response is dominated by fewer CNTs.

Apart from an interpretation based on the thermoelectric effect one may also consider quasi-metallic CNTs with a bandgap equal or close to the energy of the radiation quanta at $140 \mathrm{GHz}$ (which is about $0.5 \mathrm{meV}$ ). The response of these CNTs may be affected by direct optical transitions across the bandgap. Evidence for such transitions for quasimetallic CNTs was presented in experiments on the absorption of CNT films in the far-infrared part of the spectrum. ${ }^{19}$ In our case the asymmetry of the devices will result in different gate efficiencies for the suspended and the nonsuspended parts of a CNT the shift of the bands with respect to the Fermi level will be different. Therefore the devices we study have p-n junctions on the current path from one electrode to another at a certain range of gate voltages. Direct optical transition in the vicinity of the $\mathrm{p}$-n junction will result in the appearance of a photo-induced voltage. Fig. 3(d) explains why this may take place only in a narrow range of gate voltages close to zero. Outside of this $V_{G}$ range the Fermi levels stay either in the valence or in the conduction band of the small gap CNTs and no p-n junctions are formed. Since the frequency of the radiation corresponds to the energy of $\sim 0.5 \mathrm{meV}(\sim 5 \mathrm{~K})$ such an effect can only be observed at low temperatures and not at room temperature.

We note that DC voltage response to sub-THz radiation was observed in high-mobility $1 \mathrm{D}$ nanostructures and were ascribed to plasmonic excitations. ${ }^{20}$ In our case this scenario is not applicable since the gate electrode is not coupled directly to the radiation and high-frequency modulation of carrier density is not taking place.

Finally we discuss the responsivity of the more sensitive sample D2. The room temperature responsivity is about $2.5 \mathrm{~V} / \mathrm{W}$. While this value is not approaching those for the best available $\mathrm{THz}$ radiation detectors they are the best reported for the CNT-based devices. As stated above at room temperature the response is thermal in origin and should be affected mainly by the antenna coupling efficiency. Since the antenna used in the current work is optimized for the frequency range $0.3-3 \mathrm{THz}$ larger responsivity values may be expected for a higher THz-frequency range.

At $4.2 \mathrm{~K}$, at low modulation frequencies (below $1 \mathrm{kHz}$ ), the responsivity reaches a value of at least $500 \mathrm{~V} / \mathrm{W}$ at low power level. ${ }^{21}$ The high responsivity at $4.2 \mathrm{~K}$ is observed in a narrow gate voltage range, which makes at the moment practical applications difficult.

Due to the large impedance mismatch between the antenna and the carbon nanotube, only a small fraction of the incident power is absorbed in the nanotube. Comparison of data obtained with samples D1 and D2 indicates that decrease of device resistance may further increase the responsivity. Connecting more CNTs in parallel would improve the mismatch, but the heat conductance would also presumably increase, so we believe that devices with smaller interelectrode distance or smaller contact resistance would yield better results.

In conclusion, we have fabricated devices with CNTs, with asymmetric coupling to the thermal bath, as well as possible asymmetric doping. The devices demonstrate a strong DC voltage signal in response to radiation in the sub- $\mathrm{THz}$ range in the temperature range from 4.2 to $300 \mathrm{~K}$. The observed effect is of purely thermal origin at room temperature and may involve optical transitions over small bandgaps of quasi-metallic CNTs at low temperatures. Since the present experiments contain several different CNTs and the response is some kind of average, future experiments are needed with better defined asymmetrically mounted CNTs.

Authors thank Professor P. Barbara for providing the catalyst for CNT growth and Dr. N. Chumakov and V. Rylkov for stimulating discussions. The work was supported by the RFBR (Grant No. 12-02-01291-a) and by the Ministry of Education and Science of the Russian Federation (Contract No. 14.B25.31.0007). G.F. acknowledges support of the RFBR grant 12-02-01005-a.

${ }^{1}$ P. Avouris, M. Freitag, and V. Perebeinos, Nat. Photonics 2, 341-350 (2008).

${ }^{2}$ L. Ren, C. L. Pint, L. G. Booshehri, W. D. Rice, X. Wang, D. J. Hilton, K. Takeya, I. Kawayama, M. Tonouchi, R. H. Hauge, and J. Kono, Nano Lett. 9, 2610 (2009).

${ }^{3}$ K. Fu, R. Zannoni, C. Chan, S. H. Adams, J. Nicholson, E. Polizzi, and K. S. Yngvesson, Appl. Phys. Lett. 92, 033105 (2008).

${ }^{4}$ R. Saito, G. Dresselhaus, and M. S. Dresselhaus, Physical Properties of Carbon Nanotubes (Imperial College Press, London, 1998).

${ }^{5}$ S. M. Bachilo, M. S. Strano, C. Kittrell, R. H. Hauge, Ri. E. Smalley, and R. B. Weisman, Science 298, 2361 (2002).

${ }^{6}$ Q. Zeng, S. Wang, L. Yang, Z. Wang, T. Pei, Z. Zhang, L.-M. Peng, W. Zhou, J. Liu, W. Zhou, and S. Xie, Opt. Mater. Express 2(6), 839-848 (2012). 
${ }^{7}$ T. Mueller, M. Kinoshita, M. Steiner, V. Perebeinos, A. A. Bol, D. B. Farmer, and P. Avouris, Nat. Nanotechnol. 5, 27-31 (2010).

${ }^{8}$ C. W. Zhou, J. Kong, and H. J. Dai, Phys. Rev. Lett. 84, 5604 (2000).

${ }^{9}$ V. Zólyomi and J. Kürti, Phys. Rev. B 70, 085403 (2004).

${ }^{10}$ J. D. Chudow, D. F. Santavicca, C. B. McKitterick, D. E. Prober, and P. Kim, Appl. Phys. Lett. 100, 163503 (2012).

${ }^{11}$ M. Rinzan, G. Jenkins, H. D. Drew, S. Shafranjuk, and P. Barbara, Nano Lett. 12(6), 3097-3100 (2012).

${ }^{12}$ H. L. Hortensius, A. Öztürk, P. Zeng, E. F. C. Driessen, and T. M. Klapwijk, Appl. Phys. Lett. 100, 223112 (2012).

${ }^{13}$ B. C. St-Antoine, D. Ménard, and R. Martel, Nano Lett. 9, 3503-3508 (2009).

${ }^{14}$ A. Tselev, K. Hatton, M. S. Fuhrer, M. Paranjape, and P. Barbara, Nanotechnology 15, 1475-1478 (2004).

${ }^{15}$ S. Maslennikov, M. Finkel, S. Antipov, S. Polyakov, W. Zhang, R. Ozhegov, Y. Vachtomin, S. Svechnikov, K. Smirnov, Y. Korotetskaya, N. Kaurova, B. Voronov, and G. Gol'tsman, in Proceedings of 17th
International Symposium on Space Terahertz Technology (Paris, France, 2006), pp. 285-287.

${ }^{16}$ M. S. Dresselhaus, G. Dresselhaus, R. Saito, and A. Jorio, Phys. Rep. 409, 47-99 (2005).

${ }^{17}$ Ch. Yu, L. Shi, Z. Yao, D. Li, and A. Majumdar, Nano Lett. 5(9), 1842-1846 (2005).

${ }^{18}$ J. P. Small, K. M. Perez, and P. Kim, Phys. Rev. Lett. 91, 256801 (2003).

${ }^{19}$ T. Kampfrath, K. von Volkmann, C. M. Aguirre, P. Desjardins, R. Martel, M. Krenz, C. Frischkorn, M. Wolf, and L. Perfetti, Phys. Rev. Lett. 101, 267403 (2008).

${ }^{20}$ M. S. Vitiello, D. Coquillat, L. Viti, D. Ercolani, F. Teppe, A. Pitanti, F. Beltram, L. Sorba, W. Knap, and A. Tredicucci, Nano Lett. 12(1), 96-101 (2012).

${ }^{21}$ Reference 3 reported $50 \mathrm{~V} / \mathrm{W}$ at $4.2 \mathrm{~K}$; Ref. 12 does not state the value of responsivity, but still it can be estimated from the presented data as $5 \times 10^{4} \mathrm{~V} / \mathrm{W}$ at $4.2 \mathrm{~K}$. 